\title{
Tracking the source of cerebellar epilepsy: Hemifacial seizures associated with cerebellar cortical dysplasia
}

\author{
Agustina M. Lascano ${ }^{a}$, Alia Lemkaddem ${ }^{b}$, Cristina Granziera ${ }^{c}$, \\ Christian M. Korff ${ }^{d}$, Colette Boex ${ }^{a}$, Benoit Jenny ${ }^{e}$, \\ Thomas Schmitt-Mechelke ${ }^{f}$, Jean-Philippe Thiran ${ }^{b, g}$, Valentina Garibotto ${ }^{\text {h }}$, \\ Maria Isabel Vargas ${ }^{i}$, Karl Schaller ${ }^{\mathrm{e}}$, Margitta Seeck ${ }^{a}$, Serge Vulliemoz ${ }^{\mathrm{a}, *}$

\footnotetext{
${ }^{a}$ EEG and Epilepsy Unit, Department of Clinical Neurosciences, University Hospitals and Faculty of Medicine of Geneva, Switzerland

b Signal Processing Laboratory (LTS5), Ecole Polytechnique Fédérale de Lausanne, Switzerland

c Department of Neurology, University Hospital Center (CHUV) and University of Lausanne (UNIL), Switzerland

d Pediatric Neurology, Child and Adolescent Department, University Hospitals and Faculty of Medicine of Geneva, Switzerland

e Neurosurgery, Department of Clinical Neurosciences, University Hospitals and Faculty of Medicine of Geneva, Switzerland

f Pediatric Neurology, Children's Hospital, Luzern, Switzerland

s Department of Radiology, University Hospital Center (CHUV) and University of Lausanne (UNIL), Switzerland

${ }^{h}$ Department of Nuclear Medicine, University Hospitals and Faculty of Medicine of Geneva, Switzerland

i Department of Neuroradiology, University Hospitals and Faculty of Medicine of Geneva, Switzerland
}

Received 4 September 2012; received in revised form 29 November 2012; accepted 1 December 2012 Available online 1 February 2013

\section{KEYWORDS}

Subcortical epilepsy; Diffusion spectrum

MRI (DSI);

Electric source imaging (ESI); Hemifacial spasms; Gangliocytoma

\begin{abstract}
Summary Traditionally, subcortical structures such as the cerebellum are supposed to exert a modulatory effect on epileptic seizures, rather than being the primary seizure generator. We report a 14-month old girl presenting, since birth, with seizures symptomatic of a right cerebellar dysplasia, manifested as paroxystic contralateral hemifacial spasm and ipsilateral facial weakness. Multimodal imaging was used to investigate both anatomical landmarks related to the cerebellar lesion and mechanisms underlying seizure generation. Electric source imaging (ESI) supported the hypothesis of a right cerebellar epileptogenic generator in concordance with nuclear imaging findings; subsequently validated by intra-operative intralesional recordings. Diffusion spectrum imaging-related tractography (DSI) showed severe cerebellar structural abnormalities confirmed by histological examination. We suggest that hemispheric cerebellar lesions in cases like this are likely to cause epilepsy via an effect on the facial nuclei through ipsilateral and contralateral aberrant connections.

(c) 2013 Elsevier B.V. All rights reserved.
\end{abstract}

* Corresponding author at: EEG and Epilepsy Unit, Department of Clinical Neurosciences, University Hospitals of Geneva, 4 Rue GabriellePerret-Gentil, CH-1211 Geneva, Switzerland. Tel.: +41 2237283 52; fax: +41 223728340.

E-mail address: Serge.Vulliemoz@hcuge.ch (S. Vulliemoz). 


\section{Introduction}

The so-called "cerebellar epilepsy" is a rare and debated entity. To our knowledge, only 20 cases presenting with hemifacial seizures related to cerebellar dysplastic lesions have been reported so far. Intralesional electroencephalographic recordings (iEEG) of ictal/interictal discharges and hyperperfusion observed on ictal single photon-emission computed-tomography (SPECT) within the cerebellum support the hypothesis of a cerebellar origin of epileptic seizures (Harvey et al., 1996). We here present the case study of an infant with a cerebellar lesion and contralateral hemifacial seizures, investigated using multimodal non-invasive functional and structural imaging techniques validated with subsequent intraoperative electrophysiology.

\section{Case report}

A 14-month-old girl was referred to the epilepsy unit for the evaluation of paroxysmal hemifacial contractions. She was born after a 38-week gestation by normal uneventful vaginal delivery. From the second day of life, she presented with paroxysmal episodes starting with hyperventilation, abnormal vocalization (groaning), followed by contractions of the left orbicularis oris and orbicularis oculi muscles, drooling, fixed gaze but no nystagmus. During most episodes, right sided-facial weakness and decreased movement of the right hemibody were observed. Post-ictally, she promptly recovered level of alertness and adequate response to stimulation. These episodes occurred spontaneously at a rate of at least $10-15 /$ day during $60-180$ s and were refractory to multiple antiepileptic drugs. She had global moderate developmental delay. Neurological examination showed mild hypotonia, predominant on the right hemibody, but deep tendon reflexes were present and symmetrical. She rarely smiled and played only upon stimulation. The remaining physical examination was unremarkable.

Structural brain MRI disclosed a $2.0 \mathrm{~cm} \times 1.6 \mathrm{~cm} \times 1.9 \mathrm{~cm}$ lesion located in the superior and medial portion of the right cerebellar hemisphere, extending to the right middle and superior cerebellar peduncle, suggestive of focal dysplasia or low grade tumour (Fig. 1A). This lesion appeared hyperintense on FSE-T2-weighted and FLAIR images and was not enhanced by gadolinium injection. Long-term video-EEG monitoring showed interictal right posterior slow waves and ictal right posterior rhythmic delta slowing, but no epileptiform discharges were observed. ${ }^{18}$ FDG-PET performed during a cluster of frequent daily seizures, showed concordant "ictal" right cerebellar hypermetabolism (Fig. 1B) and ictal SPECT showed concordant hyperperfusion (Fig. 1C).

Additionally, high-resolution EEG recording was performed using a 256-electrode system. Given the absence of spikes, Electric source imaging (ESI) analysis was carried out on the EEG scalp voltage map at the $50 \%$ rising phase of the averaged right posterior slow waves $(N=12)$. A linear distributed inverse solution and the patient's individual brain were used (see Brodbeck et al., 2011 for methodological details). The maximum source was located in the right anterior quadrangular lobule of the cerebellum (Fig. 1D). In addition, we performed MRI tractography based on Diffusion Spectrum Imaging (DSI), a high angular resolution diffusion imaging technique that allows disentangling of crossing fibres and mapping of cerebellar white matter connections (Granziera et al., 2009). Technical details of acquisition and processing are detailed elsewhere (Lemkaddem et al., 2012). Tracts seeded from the middle cerebellar peduncle showed a connectivity pattern extending further than the visible lesion and affecting most of the ipsilateral cerebellar hemisphere, while climbing and parallel cerebellar fibres showed a normal anatomy in the contralateral side (Fig. 1E).

The infant underwent partial surgical resection of the lesion, sparing the middle and upper right cerebellar peduncle. Intra-operative electrophysiology included central scalp EEG, facial EMG electrodes and one depth electrode inserted into the lesion which showed pseudo-periodic $2 \mathrm{~Hz}$ epileptiform discharges. Despite lowering the level of anaesthesia, no ictal muscle activity could be recorded. Averaging of the simultaneously recorded scalp EEG centered on iEEG abnormalities showed predominantly left pericentral activity time-locked to the intralesional activity with a latency suggesting cerebello-cortical propagation (Fig. 2). Histology revealed a dysplastic gangliocytoma with a severe disruption of the cerebellar folia architecture. Post-operatively, the child quickly recovered to her pre-operative level. She has remained seizure-free for 8 months under the same antiepileptic treatment (oxcarbazepine $300 \mathrm{mg} / \mathrm{d}$ ) showing mild developmental delay (moderate impairment of verbal abilities) and EEG normalization.

\section{Discussion}

In this patient, multimodal non-invasive imaging, including ESI, with subsequent invasive electrophysiology and postoperative outcome, give strong evidence of a cerebellar seizure onset and epileptogenic zone. Severely abnormal structural connectivity supports the hypothesis that aberrant connections between the lesion and the facial nuclei might play a role in this condition.

Since initial descriptions by Langston and Tharp (1976) in the mid-1970s, only rare isolated cases have reported the association of hemifacial spasms with cerebellar lesions in infants. Similar to previous reports, ictal SPECT and PET in our patient revealed focal hyperactivity within the lesion. However, SPECT and PET are only indirect measures of neuroelectrical activity and hyperperfusion could also affect areas of seizure propagation and, moreover, hypometabolism is typically more extensive than the seizure onset zone. Therefore, the concordance with source analysis of high density EEG, for the first time in a cerebellar epileptic focus, is of particular relevance (Fig. 1D). Intralesional EEG recordings (Delande et al., 2001; Harvey et al., 1996; Yagyu et al., 2011) have been performed in some cases and have shown direct correlation between intralesional rhythmic activity and ipsilateral hemifacial seizures (Yagyu et al., 2011). Furthermore, DSI tractography, portrayed an anatomical disruption at the lesion level (Fig. 1E) and of a major cerebellar fiber bundle: the middle cerebellar peduncle (MCP), as compared to the contralateral side. However, DSI was not able to reveal the superior and inferior cerebellar peduncles when seeding from deep cerebellar nuclei in both hemispheres, as opposed to tractography results 
A

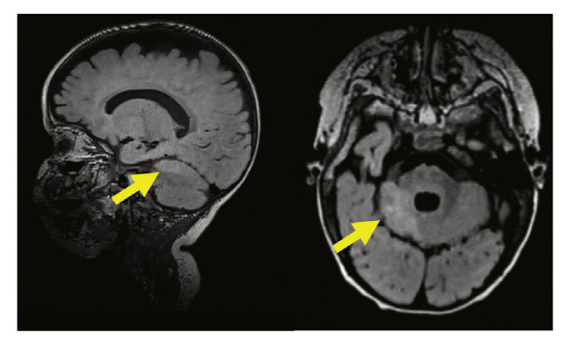

D
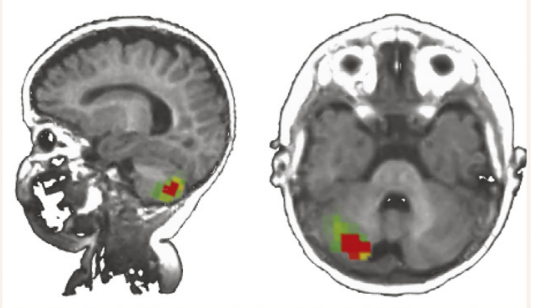

B

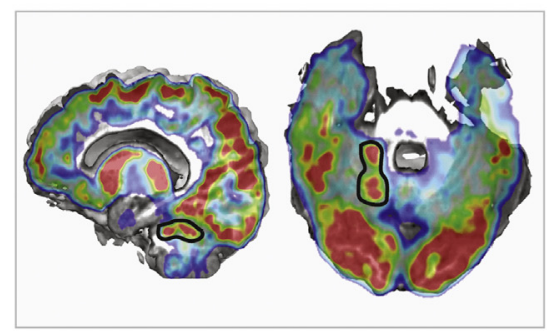

C

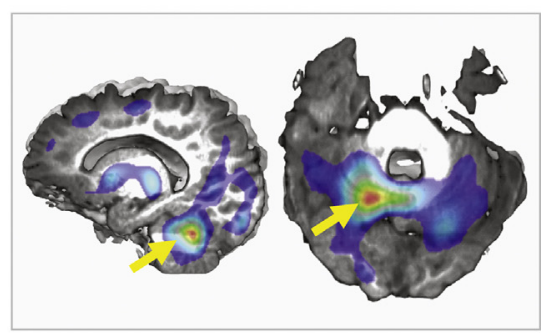

E

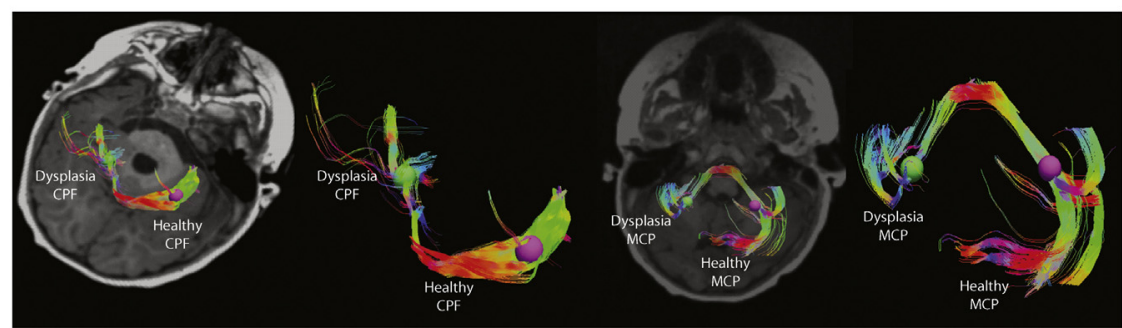

Figure 1 Non-invasive multimodal imaging. (A) Anatomical MRI showed a FLAIR hyperintensity in the superior medial portion of the right cerebellar hemisphere (yellow arrow), extending to the right middle cerebellar peduncle. (B) PET showing a right cerebellar hypermetabolism and (C) ictal SPECT showing a maximum hyperperfusion in the same area. (D) Electrical source analysis revealed a concordant localisation, within the right cerebellar hemisphere. (E) DSI tractography of cerebellar cortical parallel fibers (CPF) in the dysplastic area as well as in the healthy hemisphere overlaid on T1 image (left panel). Immediately to the right, a zoom of the obtained CPF is illustrated for the dysplastic (green seeding sphere) and the healthy hemisphere (violet seeding sphere). On the right panel, architecture of the middle cerebellar peduncle (MCP) is represented on both the dysplastic region and a corresponding region of the healthy hemisphere in a T1 weighted axial background and in a magnified version (dysplastic region is labelled by a green seeding sphere and the unaffected area with a violet seeding sphere). (For interpretation of the references to color in figure legend, the reader is referred to the web version of the article.)

obtained in healthy adults (Granziera et al., 2009). This is most likely due to the patient's immature myelination of those bundles (Ben Bashat et al., 2005; Dubois et al., 2006) and to the anatomical disruptions provoked by the large dysplastic area at the site of the lesion.

In summary, these state-of-the-art imaging techniques allowed us not only to determine the cerebellum as the most likely epileptic source in an individual case but, moreover, to assess the anatomical cytoarchitectural disorganisation of the dysplastic lesion and of major fibre bundles in vivo.

The seizure onset and symptomatogenic zones as well as the pathophysiology of such seizures related to cerebellar lesions have been largely debated in the literature, suggesting either exclusively a cerebellar role (Chae et al., 2001; Harvey et al., 1996), or a cerebellar onset with secondary spread to the brainstem (Delande et al., 2001). A recent study, using concomitant iEEG and electromyographic recordings, implied that hemifacial contractions originate in the ipsilateral cerebellar dysplastic area and propagate via the facial nucleus into the ventrolateral region of the inferior pons (Yagyu et al., 2011). In order for this to occur, aberrant cerebello-pontine connections are required as normal cerebellar output is rubro-cortical or thalamo-cortical. Interestingly, there is only one other report of hemifacial seizures contralateral to a cerebellar lesion (Dagcinar et al., 2007), all others describe ictal semiology ipsilateral to the lesion which may be due to ephaptic propagation of ictal activity.
Ictal semiology in our case consisted in a complex picture of ipsilateral weakness and contralateral dystonia, speaking against a circuit involving the primary motor cortex. The opposing bilateral facial features together with neurovegetative signs (hyperpnoea) favour abnormal cerebello-pontine/bulbar connections. There was no radiological evidence of a direct mechanical effect (compression) by the lesion on the brainstem nuclei. Previous reports of ictal hyperperfusion in both the cerebellar lesion and pons, support this hypothesis (Pontes-Neto et al., 2006). Furthermore, complete surgical resection or disconnection of the lesion result in clinical improvement with about $80 \%$ seizure freedom (Delande et al., 2001; Pontes-Neto et al., 2006). Conversely, patients with partial lesion removal did not benefit from seizure reduction after surgery (Chae et al., 2001; Park et al., 2009). Despite the fact that our patient underwent partial surgical resection, by sparing the cerebellar peduncles which were also affected, she remained seizurefree at 8 months follow-up, although a longer observation is required to better assess the outcome. To conclude, ESI and DSI tractography allow non-invasive exploration of functional and structural epileptic networks in the whole brain, even in young children. We suggest that the atypical bilateral clinical semiology, combined with ESI and DSI, could be due to abnormal structural connections between the lesion and both facial nuclei as a pathological epileptogenic neuronal network. Further improvement in brainstem tractography, with normative paediatric data would be needed to confirm this hypothesis. 


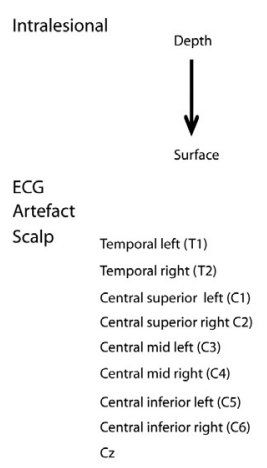

B

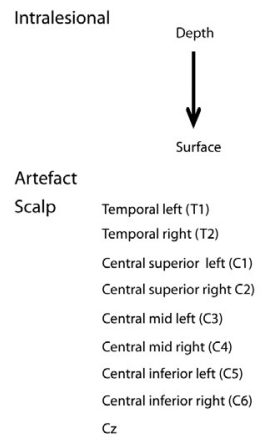

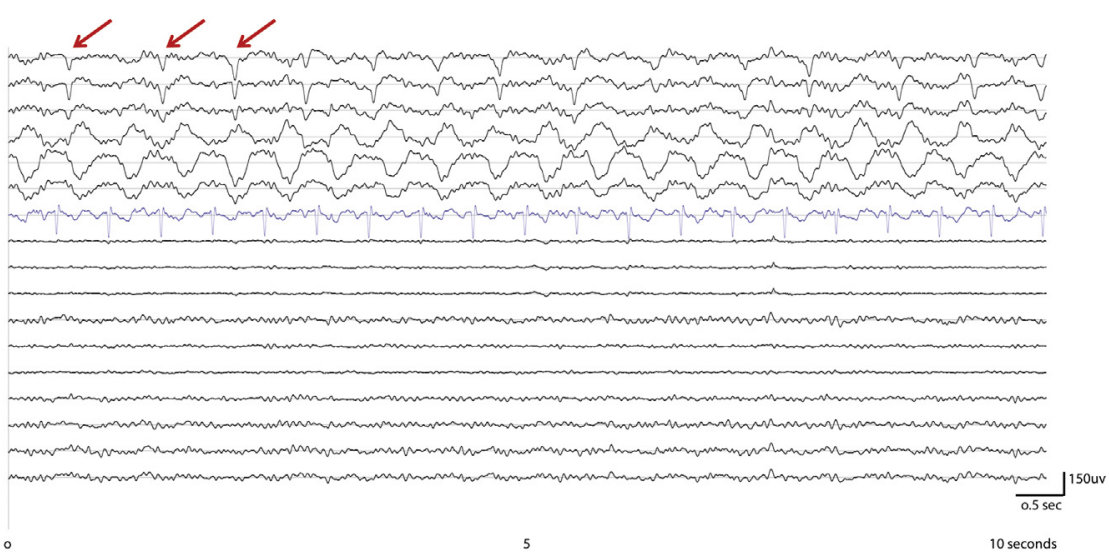

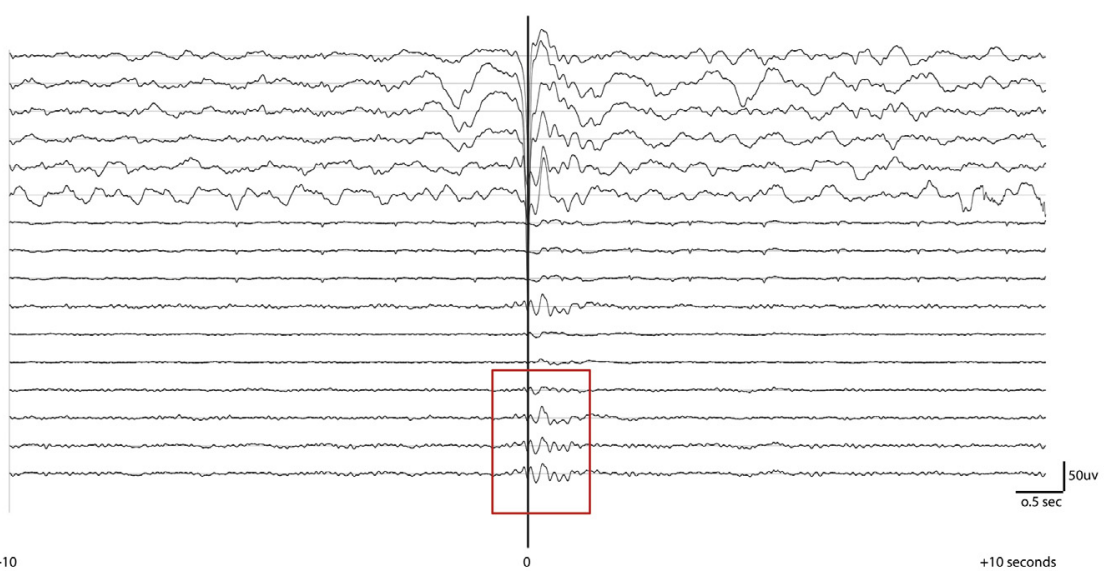

Figure 2 Intraoperative recordings. (A) Intralesional pseudo-periodic 2-2.5 Hz epileptiform discharges (red arrows). Channel 4-6 show pulse artefact time-locked to ECG. (B) Average of the simultaneously recorded scalp EEG centered on the intracranially recorded epileptiform discharges $(N=660)$ reveals a left pericentral activity (red rectangle). (For interpretation of the references to color in figure legend, the reader is referred to the web version of the article.)

\section{Disclosure of conflict of interest}

MS and SV received speaker fees from Electric Geodesic Inc. None of the other authors has any conflict of interest to disclose. We confirm that we have read the Journal's position on issues involved in ethical publication and affirm that this report is consistent with those guidelines.

\section{Acknowledgements}

Work supported by Swiss National Science Foundation grants FNS 140332 (SPUM Epilepsy) and grants 141165, 144529, 122073. The authors wish to thank Remi Tyrand for technical assistance during intralesional recordings and $\mathrm{Dr}$. Laurent Spinelli for image coregistration. High resolution electroencephalogram analysis was performed by means of Cartool software (http://brainmapping.unige.ch/Cartool.php). This software is developed by Denis Brunet, from the Functional Brain Mapping Laboratory, Geneva, supported by the Center for Biomedical Imaging (CIBM), Geneva and Lausanne, Switzerland.

\section{References}

Ben Bashat, D., Ben Sira, L., Graif, M., Pianka, P., Hendler, T., Cohen, Y., Assaf, Y., 2005. Normal white matter development from infancy to adulthood: comparing diffusion tensor and high $\mathrm{b}$ value diffusion weighted MR images. J. Magn. Reson. Imaging 21, 503-511.

Brodbeck, V., Spinelli, L., Lascano, A.M., Wissmeier, M., Vargas, M.I., Vulliemoz, S., Pollo, C., Schaller, K., Michel, C.M., Seeck, M., 2011. Electroencephalographic source imaging: a prospective study of 152 operated epileptic patients. Brain 134, 2887-2897.

Chae, J.H., Kim, S.K., Wang, K.C., Kim, K.J., Hwang, Y.S., Cho, B.K., 2001. Hemifacial seizure of cerebellar ganglioglioma origin: seizure control by tumor resection. Epilepsia 42, 1204-1207.

Dagcinar, A., Hilmi Kaya, A., Ali Tasdemir, H., Kuruoglu, E., Sabancilar, Z., Sav, A., 2007. A fourth ventricular ganglioneurocytoma representing with cerebellar epilepsy: a case report and review of the literature. Eur. J. Paediatr. Neurol. 11, 257-260.

Delande, O., Rodriguez, D., Chiron, C., Fohlen, M., 2001. Successful surgical relief of seizures associated with hamartoma of the floor of the fourth ventricle in children: report of two cases. Neurosurgery 49, 726-730 (discussion 730-721).

Dubois, J., Hertz-Pannier, L., Dehaene-Lambertz, G., Cointepas, Y., Le Bihan, D., 2006. Assessment of the early organization and maturation of infants' cerebral white matter fiber bundles: a 
feasibility study using quantitative diffusion tensor imaging and tractography. Neuroimage 30, 1121-1132.

Granziera, C., Schmahmann, J.D., Hadjikhani, N., Meyer, H., Meuli, R., Wedeen, V., Krueger, G., 2009. Diffusion spectrum imaging shows the structural basis of functional cerebellar circuits in the human cerebellum in vivo. PLoS ONE 4, e5101.

Harvey, A.S., Jayakar, P., Duchowny, M., Resnick, T., Prats, A., Altman, N., Renfroe, J.B., 1996. Hemifacial seizures and cerebellar ganglioglioma: an epilepsy syndrome of infancy with seizures of cerebellar origin. Ann. Neurol. 40, 91-98.

Langston, J.W., Tharp, B.R., 1976. Infantile hemifacial spasm. Arch. Neurol. 33, 302-303.

Lemkaddem, A., Daducci, A., Vulliemoz, S., O’Brien, K., Lazeyras, F., Hauf, M., Wiest, R., Meuli, R., Seeck, M., Krueger, G., Thiran, J.P., 2012. A multi-center study: intra-scan and inter-scan variability of diffusion spectrum imaging. Neuroimage 62, 87-94.

Park, Y.S., Oh, M.C., Kim, H.D., Kim, D.S., 2009. Early surgery of hamartoma of the floor of the fourth ventricle: a case report. Brain Dev. 31, 347-351.

Pontes-Neto, O.M., Wichert-Ana, L., Terra-Bustamante, V.C., Velasco, T.R., Bustamante, G.O., Fernandes, R.M., AzevedoMarques, P.M., Oliveira, L.F., Santos, A.C., Kato, M., Inuzuka, L.M., Machado, H.R., Sakamoto, A.C., 2006. Pontine activation during focal status epilepticus secondary to hamartoma of the floor of the fourth ventricle. Epilepsy Res. 68, 265-267.

Yagyu, K., Sueda, K., Shiraishi, H., Asahina, N., Sakurai, K., Kohsaka, S., Sawamura, Y., Saitoh, S., 2011. Direct correlation between the facial nerve nucleus and hemifacial seizures associated with a gangliocytoma of the floor of the fourth ventricle: a case report. Epilepsia 52, e204-e206. 